\title{
Corrosion resistance of thermal diffusion zinc coatings of "PNTZ" in oilfield environments
}

\author{
Aleksandr Biryukov ${ }^{1, *}$, Dmitry Zakharyevich ${ }^{2}$, Rashit Galin $^{2}$, Natalya Devyaterikova ${ }^{3}$, Igor Scherbakov $^{3}$ \\ ${ }^{1}$ Chelyabinsk State University, 454001 Chelyabinsk, Br. Kashirinykh str. 129, Russian Federation \\ ${ }^{2}$ LLC VIKA-GAL, 454138 Chelyabinsk, Molodogvardeytsev str. 7, Russian Federation \\ ${ }^{3}$ JSC Pervouralsk New Pipe Plant, 623112, Pervouralsk, Torgovaya str. 1, Russian Federation
}

\begin{abstract}
The production, composition, wear resistance and corrosion resistance of thermal diffusion zinc coatings produced at JSC PNTZ are considered. Specially treated zinc powders with nanocrystallized oxide on the surface of the particles are used in production. The process is capable to provide unique composition and microstructure of coatings. The results of laboratory and field tests show excellent characteristics of coatings in conditions typical for the oil industry.
\end{abstract}

\section{Introduction}

In 2008-2010, JSC Pervouralsk New Pipe Plant (JSC "PNTZ"), together with LLC VIKA-GAL, developed and implemented equipment and technology for thermal diffusion galvanizing (TDG) of couplings for tubing and casing pipes. A distinctive feature of the technology is the use of zinc powder with nanostructured oxide on the surface of particles without any additives. Investigations of coatings obtained using such powders have shown technological capability for obtaining a great variety of phase and dispersion compositions of coatings in comparison with traditional thermal diffusion zinc coatings. The motivation for the introduction of technology was the anti-seize properties of such coatings. Yet, the predominant use of zinc coatings is corrosion protection of steels. Corrosion in the oilfield environments is often enhanced by hydro-abrasive wear. To alleviate this problem, a combination of corrosion resistance and appropriate mechanical properties is needed. The technical ability of the process to produce coatings of different composition makes it possible to create the necessary combination of properties for the protection of steel products under oil production conditions. In this article, we review the results of laboratory and field tests of corrosion and wear behavior of thermal diffusion coatings produced on "PNTZ".

\section{Process}

The method of thermal diffusion galvanizing (TDG) has a number of advantages over other methods of zinc coating preparation, but its capabilities are not fully utilized due to insufficient information about the properties of the coating. TDG coatings provides the best corrosion characteristics, being at the same time less energy-consuming and dangerous to the environment comparing with electroplating and hot-dip galvanizing[1,2].

The process implemented on "PNTZ" uses zinc powder specially treatedaccording to [3], without traditional additives such as inert components and halide activators. The powders have a composition of $90 \% \mathrm{Zn}$ metal and $10 \% \mathrm{ZnO}$, the latter is in the form crystals with nanosized cross section on the surface of metal core, in contrast to usual compact dense oxide film (Fig.1). The difficulty of condensation of zinc vapour on the surface of zinc oxide nanocrystals eliminates the use of inert fillers, and pores with dimensions in nanometer range between the crystals contribute to accelerated mass transfer of zinc to the surface of the steel productdue to capillary action. Single component zinc powder-based mixture with nanocrystallized particles surface does not require replacement in the process of its long-term application in closed cycle.

Thermal diffusion galvanizing of couplings is carried out by heat treatment of products in a rotating retort (with reverse movement) in specially treated zinc powder with heating of the furnace to a temperature in the range of $380-420^{\circ} \mathrm{C}$.

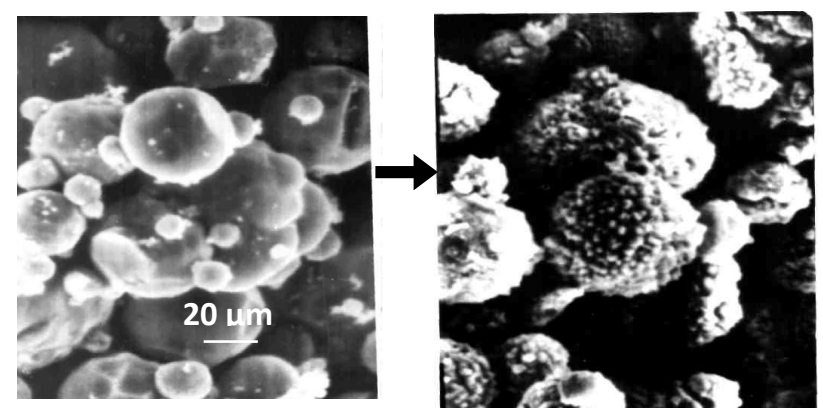

Fig. 1.Zinc powder transformation during special (hydrothermal) treatment according to [3]: SEM images of the particles of original powder (left) and powder after treatment (right). Equal scales on both images.

\footnotetext{
*Corresponding author: st4857@yandex.ru
} 


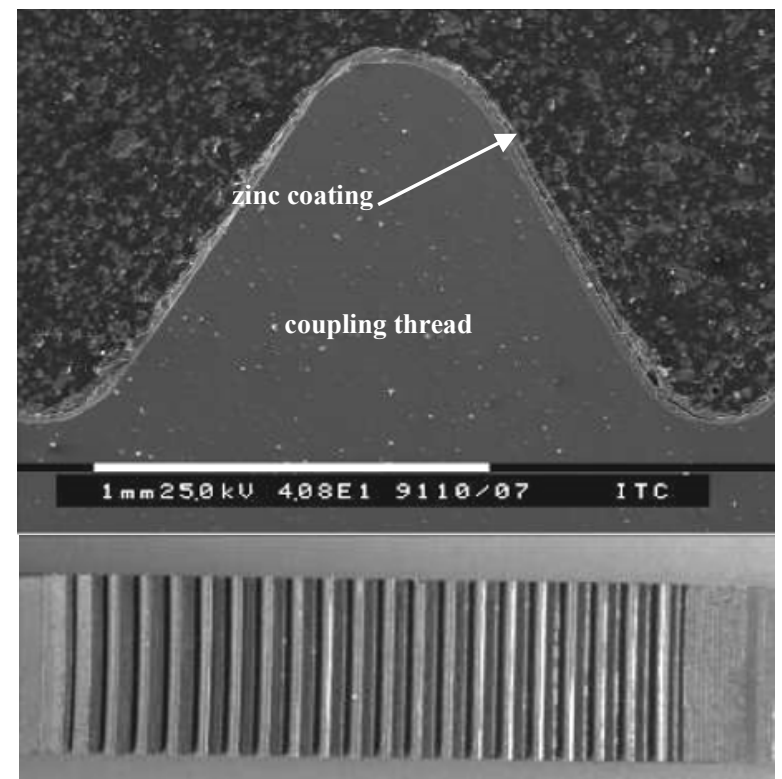

Fig. 2.SEM image ofthe cross section (top) and the appearance (bottom) of coupling thread after TDG

\section{Composition of coatings}

The process allows formation of uniform coating at coupling threaded surface with exact reproduction of thread and sealing elements profile (Fig.2), ensuring the high air tightness of threaded connections pipe-coupling.

In contrast to the hot-dip galvanizing coatings, the thermal diffusion zinc coating consists entirely of intermetallic $\mathrm{Fe}-\mathrm{Zn}$ phases. These phases have different physico-chemical properties, and the ability to change the proportion of these phases allows flexible control of the properties of the coating. Figure 3 shows typical X-

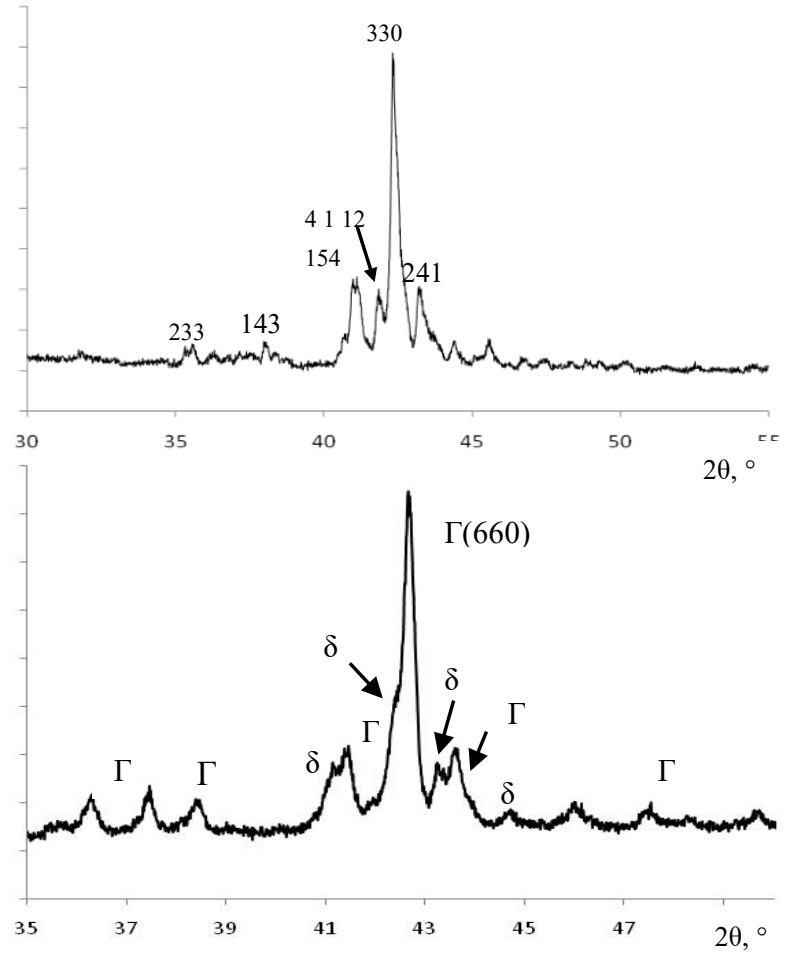

Fig. 3. X-ray diagrams of the coatings with total thickness $25 \mu \mathrm{m}$ (single $\delta$ phase, top) and $15 \mu \mathrm{m}$ (bottom). ray diagrams of the coatings produced on "PNTZ". Depending on the temperature regime and the couplings/powder volume ratio, there are either peaks of the $\delta$ phase $\left(\mathrm{FeZn}_{7-10}\right)$ only, or a superposition of peaks from the $\delta$ and $\Gamma\left(\mathrm{Fe}_{3} \mathrm{Zn}_{10}\right)$ phases.

The study of coating cross sections with a total thickness of $25 \mu \mathrm{m}$ (Fig. 4, top) shows that a single $\delta$ phase layer occupies the top $10 \mu \mathrm{m}$ of the coating, the next $10 \mu \mathrm{m}$ correspond to the coexistence region of the $\delta$ and $\Gamma$ phases, the single $\Gamma$ phase layer occupies about 5 $\mu \mathrm{m}$ from the steel base. A more detailed study of the transition mixed layer showed the presence of nanocrystalline precipitates, which could be assigned to $\Gamma$ phase (Fig.4, bottom). The $\delta$ phase is usually considered to be relatively ductile, playing an important role in deformation of the coating during processing and operation [4]. On the other hand, the $\Gamma$ phase is hard and brittle. This fine-dispersed coating structure with nanoinclusions can simultaneously provide high hardness and ductility and contribute to improving the wear resistance of the coating.

\section{Wear resistance}

Operation of pilot batches of tubing with couplings with thermal diffusion zinc coatingproduced on "PNTZ" showed excellentanti-seize performance. The pipes with TDG couplings withstood up to $70 \mathrm{RIH} / \mathrm{POOH}$ operations in comparison to $19 \mathrm{RIH} / \mathrm{POOH}$ typical for traditional TDG, with a minimum change in the

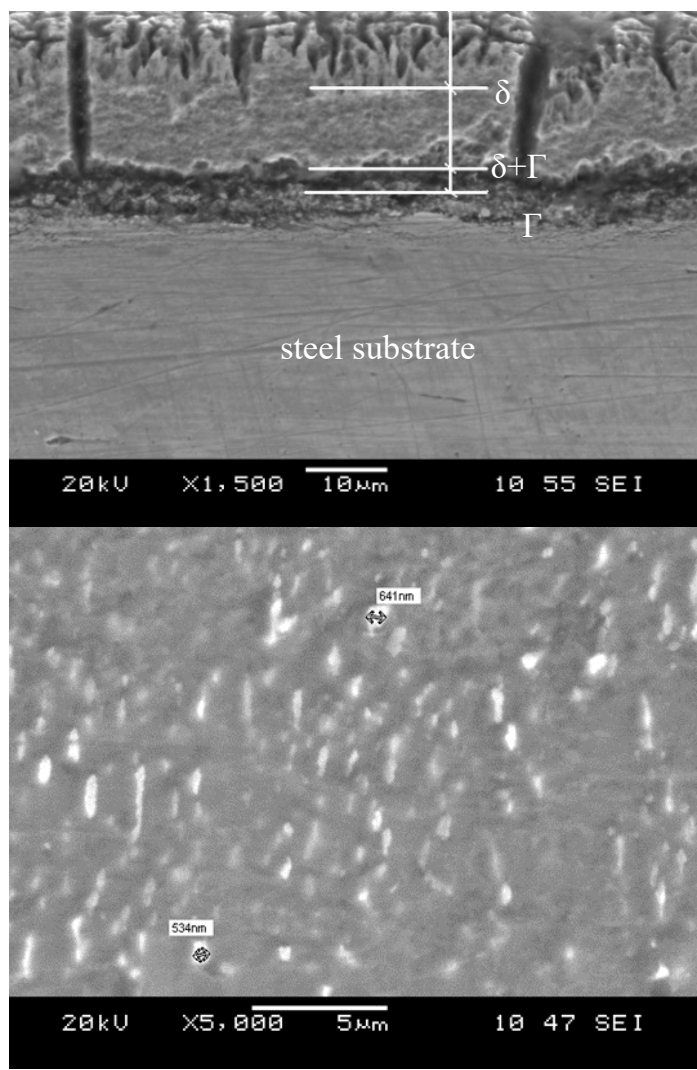

Fig. 4.Microstructure of the coating of a thickness $25 \mu \mathrm{m}$ : SEM image of the cross section with phase boundaries determined by EDX (top) and detailed image of transition $\delta+\Gamma$ region (bottom). 
geometrical parameters of the threaded joint and a $50 \%$ reduction in the thickness of the zinc layer during control tests.

Laboratory tests for wear on TDG coatings in accordance with ASTM G 65-94 showed nonmonotonous wear.To clarify the causes of this behavior, the time dependences of the wear rate were compared with the results of chemical microanalysis of cross sections of the samples (Fig. 5). The rate of wear of the $\delta$-phase layer in the thermal diffusion zinc coating decreases with decreasing zinc content in the layer, i.e. when approaching the substrate. The wear rates of the upper and lower boundaries of the $\delta$-phase in the coating differ by 4-5 times. Local maximum of the wear rate occurs near the phase boundary between $\delta$ and $\Gamma$, after which the wear rate continues to fall. The highest wear rates are observed at the beginning of the test. At the same time, the transfer of the coating to the steel counterbody was visually observed. Intensive wear of thick coatings can be attributed to the presence of an extensive system of grain boundaries in such coatings, along which microcrack development and layer destruction occur. The formation of a layer of zinc coating on the surface of the steel in contact with it causes the wear in this pair to become uniform. This explains the slow uniform wear of the threaded connection of the pipe-coupling without the seizing, which allowed to multiply the number of $\mathrm{RIH} / \mathrm{POOH}$ ("screwing-unscrewing") operations. Exclusive combination of high density and at the same time plasticity ensuring hard lubrication effect, thickness uniformity and strict duplication of threaded surface profile significantly increased operation reliability of tubing and increased operation time.

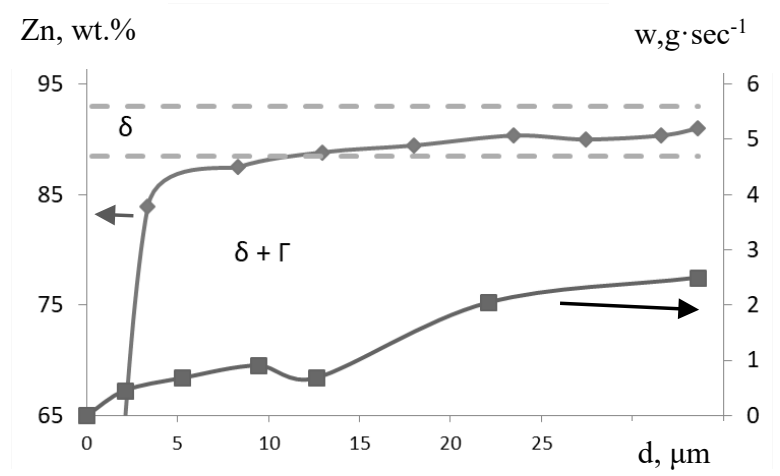

Fig. 5. Zinc content (on the left) and wear rate (on the right) of the TDG coating as a function of abrasion depth.

\section{Corrosion}

\subsection{Laboratory corrosion test}

The coating samples were immersed for 720 hours in 3 wt. $\% \mathrm{NaCl}$ solutions with $\mathrm{pH}$ from 3 to 7 . After immersion the samples were studied by X-ray diffraction analyses and microscopic methods. X-ray difraction shows that the main phase of the coating persists after 720 hours of contact with both neutral and weakly acidic corrosive media. On the SEM image of the coating after corrosion in a medium with $\mathrm{pH} 3$, it can be seen that part of the coating has survived despite the aggressiveness of the medium.

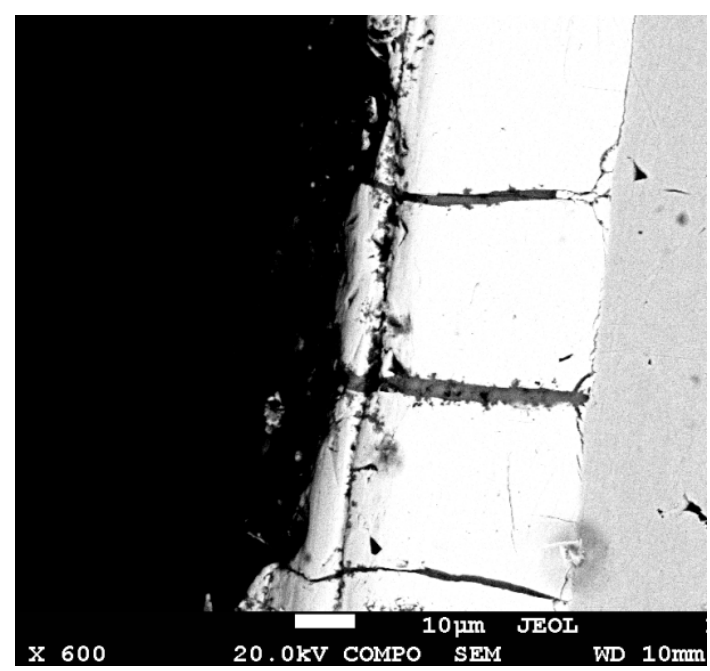

Fig. 6. SEM image of TDZ coating after corrosion in environment with $\mathrm{pH} 3$.

Corrosion products penetrate the cracks and cover the areas of the dissolved coating. This prevents the coating from further dissolving. According to the data of X-ray diffraction and chemical microanalyses, the corrosion products of TDZ coatings are simonkolleite and hydrozincite.

\subsection{Oilfield corrosion test}

Steel couplings with TDG coatings $(40 \mu \mathrm{m})$, manufactured by "PNTZ", were exposed for 120 days in an oil well in a high $\mathrm{CO}_{2}$ content, chloride ion content of 23-24 g/l and small amounts of $\mathrm{H}_{2} \mathrm{~S}$. Average pressure is $240 \mathrm{~atm}$, temperature is up to $85^{\circ} \mathrm{C}$.After extraction from the well, the pipes are examined for corrosion damage visually and by means of metallography of cross sections. Corrosion products of TDG coatings were studied by X-ray diffraction analysis, the chemical composition was determined by scanning electron microscopy with EDX analyses.

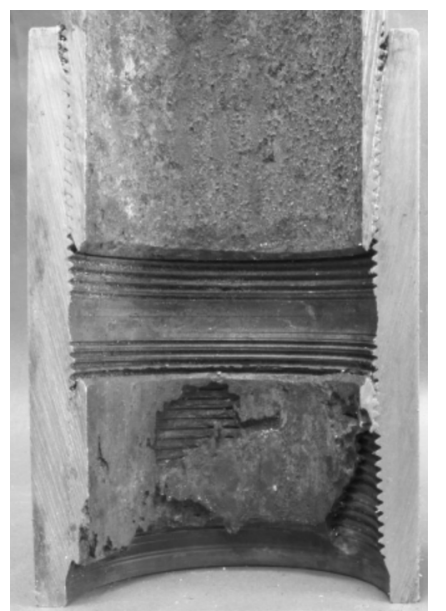

Fig. 7. The appearance of the coupling after corrosion. 
Visual inspection of a threaded joint with a galvanized coupling after exposure to the connecting pipes shows strong penetration corrosion, while only a few points of pitting corrosion with a depth of less than $0.1 \mathrm{~mm}$ are noted on the coupling. Corrosion products of connected pipes are friable, light brown in color. On the surface of the coupling a layer of corrosion products is uniform, dense, dark brown. The cross section of the inter-thread partwas made for further studies. Examination by scanning electron microscopy shows that the coating is retained at TDG coupling thread. At the same time, between the threads, the coating with a thickness of about $40 \mu \mathrm{m}$ is completely replaced by dense layers of corrosion products with a thickness of 20-30 $\mu \mathrm{m}$ (Fig.8)

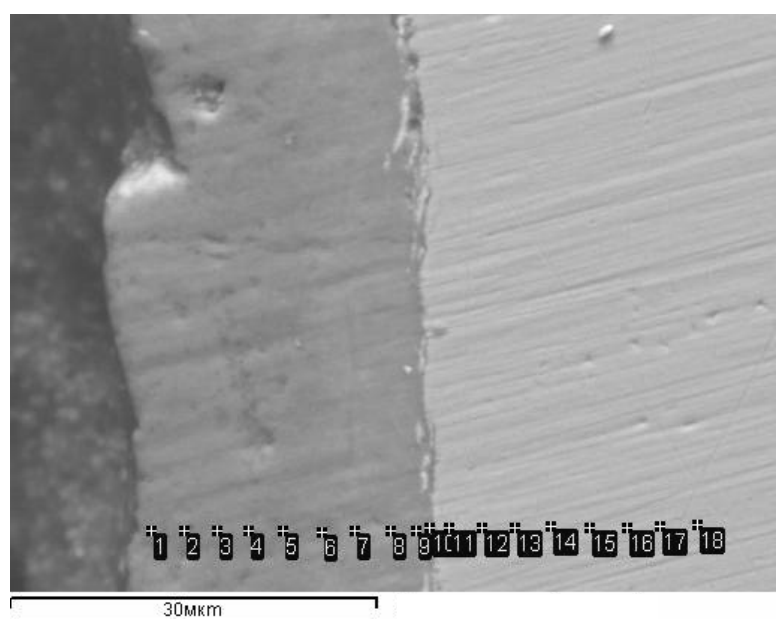

Fig. 8. SEM image layer of corrosion products.

The content of zinc and iron in the layer of corrosion products increases when approaching from the surface to the base metal. The zinc content increases from 20 to 30 at. $\%$, and iron from 4 to 12 at. $\%$ The amount of oxygen, on the contrary, decreases from 75 to 60 at. \% Also, a small amount of calcium and sulfur was found on the surface, apparently due to chemical precipitation from the medium and the effect of dissolved hydrogen sulfide. The increase in the content of zinc and iron in the direction to the base metal in the layer of corrosion products indicates the diffusion nature of the formation of corrosion products. According to X-ray diffractionanalyses, the corrosion products are mixed carbonates of iron and zinc.

\section{Conclusions}

1. Thermal diffusion zinc coatings produced by JSC "PNTZ" simultaneously satisfy the requirements of repeated "screwing - unscrewing" of threaded joints and corrosion resistance in oil wells with highly corrosive media.

2. Exclusive combination of high density and at the same time plasticity ensuring hard lubrication effect, thickness uniformity and strict duplication of threaded surface profile significantly increased operation reliability of tubing and increased operation time
3. Thermal diffusion zinc coatings produced by JSC "PNTZ" have a low corrosion rate both in neutral and in weakly acidic media, in contrast to pure zinc.

4. The high corrosion resistance of the coatings is associated with the formation of dense layers of corrosion products that protect the base metal.

\section{References}

1. F. Natrup, W. Graf, Thermochemical Surface Engineering of Steels (Woodhead Publishing, 2015), 737

2. A. Schmitz, H. Bracht, F. Natrup, W. Graf, International Heat Treat. Surf. Eng. 2, 49 (2008)

3. R.G. Galin, RU Patent 2170643 (2001)

4. A.R. Marder, Prog. in Mater. Sci. 45, 191 (2000) 